\title{
Holographic Metalens for Switchable Focusing of Surface Plasmons
}

\section{Citation}

Wintz, Daniel, Patrice Genevet, Antonio Ambrosio, Alex Woolf, and Federico Capasso. 2015.

"Holographic Metalens for Switchable Focusing of Surface Plasmons." Nano Letters 15 (5): 3585-89. https://doi.org/10.1021/acs.nanolett.5b01076.

\section{Permanent link}

http://nrs.harvard.edu/urn-3:HUL.InstRepos:41371319

\section{Terms of Use}

This article was downloaded from Harvard University's DASH repository, WARNING: This file should NOT have been available for downloading from Harvard University's DASH repository.

\section{Share Your Story}

The Harvard community has made this article openly available.

Please share how this access benefits you. Submit a story.

\section{Accessibility}




\title{
Holographic Metalens for Switchable Focusing of Surface Plasmons
}

\author{
Daniel Wintz, ${ }^{\dagger}$ Patrice Genevet, ${ }^{\dagger, \dagger}$ Antonio Ambrosio, ${ }^{\dagger, \S}$ Alex Woolf, ${ }^{\dagger}$ and Federico Capasso ${ }^{\dagger}{ }^{\dagger}$ \\ ${ }^{\dagger}$ School of Engineering and Applied Sciences, Harvard University, 9 Oxford Street, McKay 125, Cambridge, Massachusetts 02138 \\ United States \\ ${ }^{*}$ Singapore Institute of Manufacturing Technology, 71 Nanyang Drive, Singapore 638075, Singapore \\ ${ }^{\S}$ CNR-SPIN U.O.S. Napoli, Dipartimento di Fisica, Università di Napoli Federico II, Complesso Universitario di Monte Sant’Angelo, \\ Via Cintia, 80126 - Napoli, Italy
}

\section{Supporting Information}

ABSTRACT: Surface plasmons polaritons (SPPs) are lightlike waves confined to the interface between a metal and a dielectric. Excitation and control of these modes requires components such as couplers and lenses. We present the design of a new lens based on holographic principles. The key feature is the ability to switchably control SPP focusing by changing either the incident wavelength or polarization. Using phase-sensitive near-field imaging of the surface plasmon wavefronts, we have observed their switchable focusing and steering as the wavelength or polarization is changed.

KEYWORDS: Metasurfaces, surface plasmons, holography, metalens
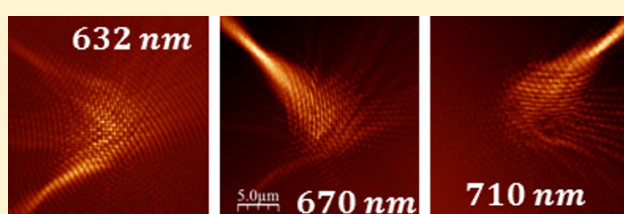

\section{$750 \mathrm{~nm}$}

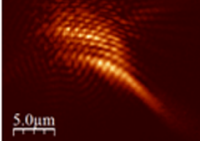

S urface plasmon polaritons (SPPs) are electromagnetic surface waves confined to propagate at the interface of a metal and a dielectric, involving both a charge oscillation of free electrons in the metal and an evanescent electric field extending into the dielectric. ${ }^{1}$ SPPs have garnered attention for decades now, affording the possibility to control light at the nanoscale, transfer information on subwavelength scales, and other novel optical techniques. $^{2-4}$ Coupling into SPP modes can be accomplished by simple experimental configurations, typically by using prism geometries or diffractive gratings. In addition, the development of high-resolution lithography and milling techniques allows for the design of structures that can be integrated into complex optoelectronic circuits whose functionality is based on the processing of SPPs. Recently developed nanostructured surfaces (metasurfaces) that control the amplitude, phase, and polarization of propagating light or SPPs are particularly suited for wavefront engineering. ${ }^{5-9}$ Metasurfaces based on plasmonics have already been used to implement flat lenses that work in transmission ${ }^{10-13}$ or in reflection $^{14,15}$ with reduced aberrations. ${ }^{16,17}$ On the metaldielectric interface, wavelength selective focusing ${ }^{18,19}$ and unidirectional coupling to plane waves ${ }^{20}$ have also been shown by means of plasmonic couplers made of nanoslits. Nanoholes and nanoslits are among the most commonly used unit elements when designing metasurfaces for SPP manipulation. $^{21-24}$

Here, we demonstrate a metalens design strategy based on holographic principles. The metalens has the ability to steer SPPs between several foci on the surface based on the incident wavelength and has on/off switchability based on the incident polarization. Our design is straightforward and consists of placing nonresonant nanoslits (50 nm wide, $200 \mathrm{~nm}$ long) along the contours defined by the wavefront of SPPs propagating out from an imaginary emitter placed at the location on the surface where we want SPP focusing. The imaginary point source is treated as a Huygens' emitter that can be described by a simple $2 \mathrm{D}$ circular wave: $\vec{E} \propto e^{i k_{\mathrm{SPP}} \cdot r}$, where $k_{\mathrm{SPP}}$ is the wavevector of the SPP at gold/air interface and $r$ is the distance from the imaginary source point (Figure 1). Because the nanoslits lie on the equiphase lines (wavefront of the SPPs), if the nanoslits are excited in phase then the reradiated SPPs will constructively interfere at the position of the original imaginary emitter. Because we want to have multiple foci (for tunability purposes), adding another focus requires the nanoslits to be placed not just on the equiphase lines of one imaginary emitter but on the equiphase points resulting from the intersections of the wavefronts of all the imaginary point sources (Figure 1). In our device, a different source point for each free space wavelength $(632,670,710$, and $750 \mathrm{~nm})$ is chosen such that light at each wavelength couples to SPPs via the nanoslits and is focused to the four corners of a $16 \mu \mathrm{m} \times 16$ $\mu \mathrm{m}$ square. In Figure $1 \mathrm{a}, \mathrm{b}$, the mutual wavefront intersections are denoted by the black dots, which are the positions where we mill nanoslits in the gold film.

Interestingly, our approach is a special case of a more general design principle based on holography, ${ }^{25-27}$ which has been extended for use with surface plasmons. ${ }^{28-37}$ In general, holography involves recording the interference pattern between a suitably chosen reference wave and the light scattered by an object into a photosensitive material. Both intensity and phase

Received: March 18, 2015

Revised: April 23, 2015

Published: April 27, 2015 
a)

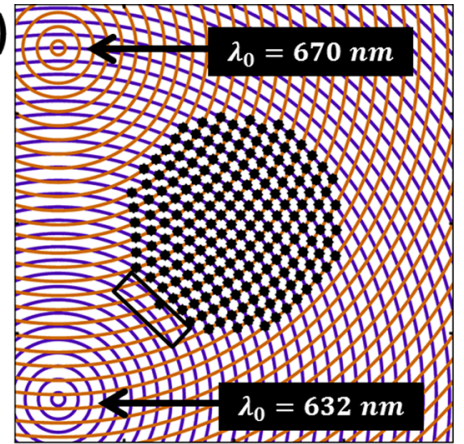

b)

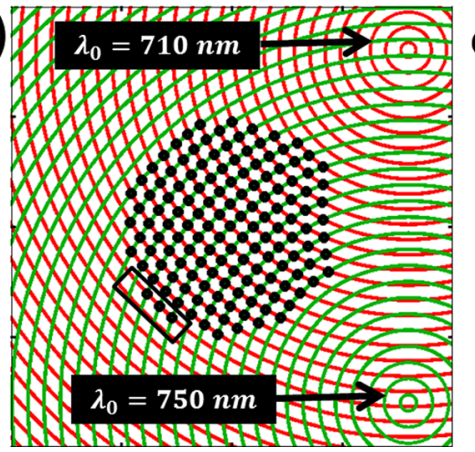

c)

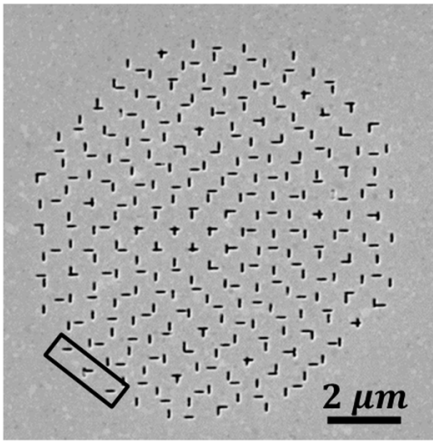

Figure 1. Metalens design. (a) Focal points for illuminating light wavelengths $\lambda_{0}=632$ and $670 \mathrm{~nm}$ in the bottom and top left corners, respectively. Purple circles denote the wavefronts of an imaginary circular point source located in the bottom left corner for $\lambda_{0}=632 \mathrm{~nm}\left(\lambda_{\mathrm{SPP}}=604 \mathrm{~nm}\right)$. Orange circles denote the wavefront for $\lambda_{0}=670 \mathrm{~nm}$ imaginary source in the top left corner. Black dots denote the intersections of the two wavefronts (within a $5 \mu \mathrm{m}$ radius), which is where vertical nanoslits are milled. Vertical nanoslits couple light into surface plasmon polaritons preferentially when excited with normally incident horizontally polarized light. Thus, if the vertical apertures are illuminated with the latter at $\lambda_{0}=632 \mathrm{or} 670 \mathrm{~nm}$ light, all of the scattered surface plasmons that reach the respective focal point (on the left side) will be in phase because by design they lie on the equiphase lines. (b) Equivalent of panel a but for the other two wavelengths $\left(\lambda_{0}=710\right.$ and $\left.750 \mathrm{~nm}\right)$, which will be focused to the remaining corners of the $16 \mu \mathrm{m} \times 16 \mu \mathrm{m}$ square. Black circles in panel $\mathrm{b}$ denote where horizontal nanoslits are milled. There are fewer intersection (equiphase) points because the wavelengths are longer. (c) Scanning electron micrograph of our device after the nanoslits are etched into the gold film by focused ion beam. Even though some of the nanoslits overlap, this does not appreciably affect the performance of the device. The black box is shown for reference and denotes the same physical space in all panels.

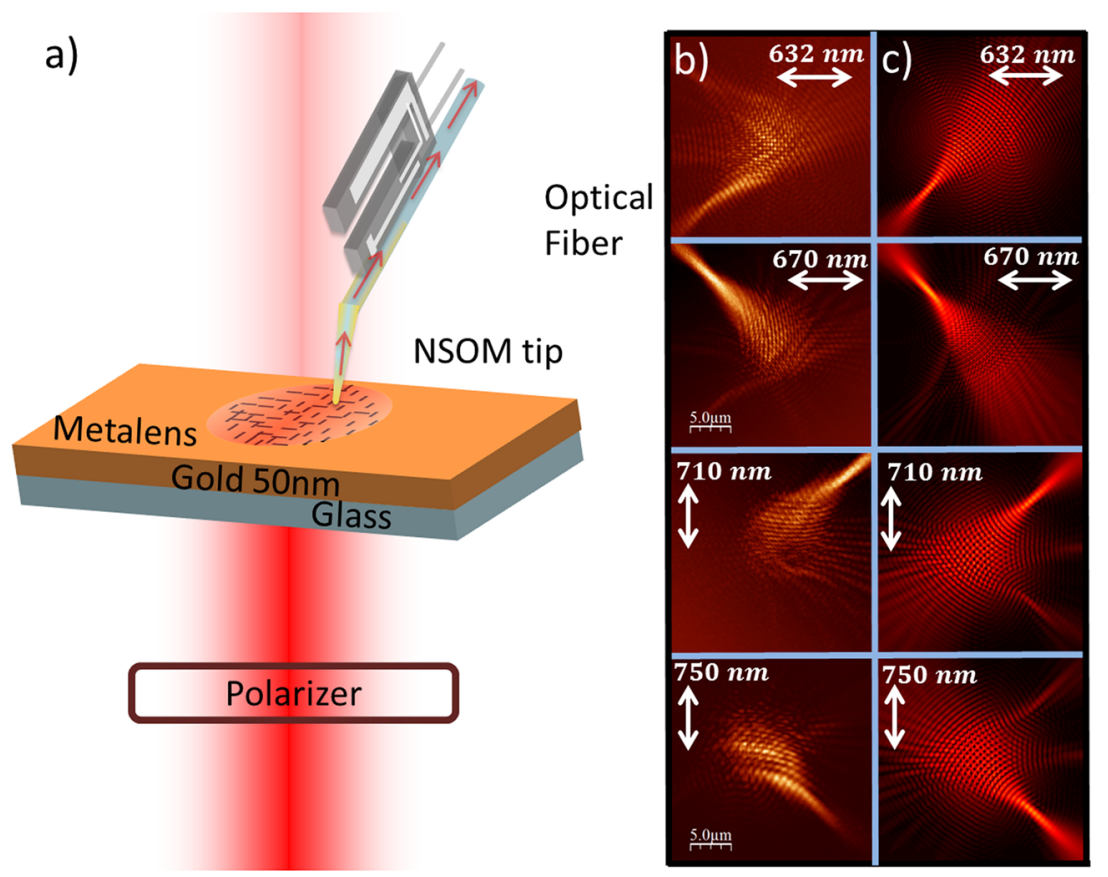

Figure 2. Experimental setup and results. (a) Experimental setup; light from a supercontinuum laser source is incident on the sample from below. Light at a single wavelength is passed through a polarizer in order to set the incident light polarization, and the light is focused onto the metalens (not depicted). Some of the laser light is transmitted, which can produce an interference pattern in the data depending on the relative intensities of the SPPs and the transmitted light. NSOM is used to collect light. The NSOM tip, which is a metal-coated, tapered optical fiber, interacts with the evanescent field of the SPP and scatters it, converting energy from the SPP mode into a propagating waveguide mode in the optical fiber. (b) Experimental results with the polarization of light denoted by the white arrow. SPP beams are unidirectionally focused to the four corners of a square depending on the wavelength and polarization. (c) Analytical simulations used to compare to the experimental results. Each aperture (with the correct orientation) in the metalens is treated as a point dipole and the fields from all the nanoslits are summed to give the in-plane total electric field.

information on the object's scattered wavefront can be retrieved by illuminating the recorded interference pattern with the reference beam. In regards to our device, the equiphase lines (on which the nanoslits are placed) are equivalent to the lines of constructive interference between a flat wavefront (e.g., a normally incident plane wave) and a circular point source, where the flat wavefront assumes the holographic role of the suitably chosen reference wave and the SPP from the imaginary point source assumes the role of the light scattered by an object. It is worth noting here that an iterative algorithm is not needed; ${ }^{18,38}$ most holographic approaches utilize such algorithms to maximize the constructive interference between antennas. Moreover, as these algorithms only aim to maximize the intensity at a specific position, they do not necessarily result 

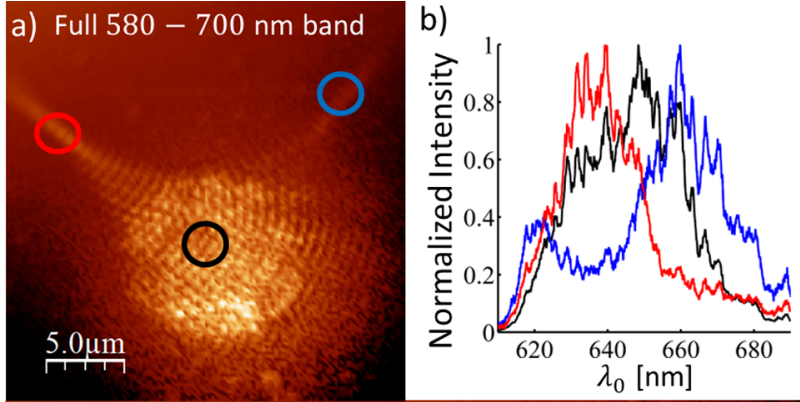

e)
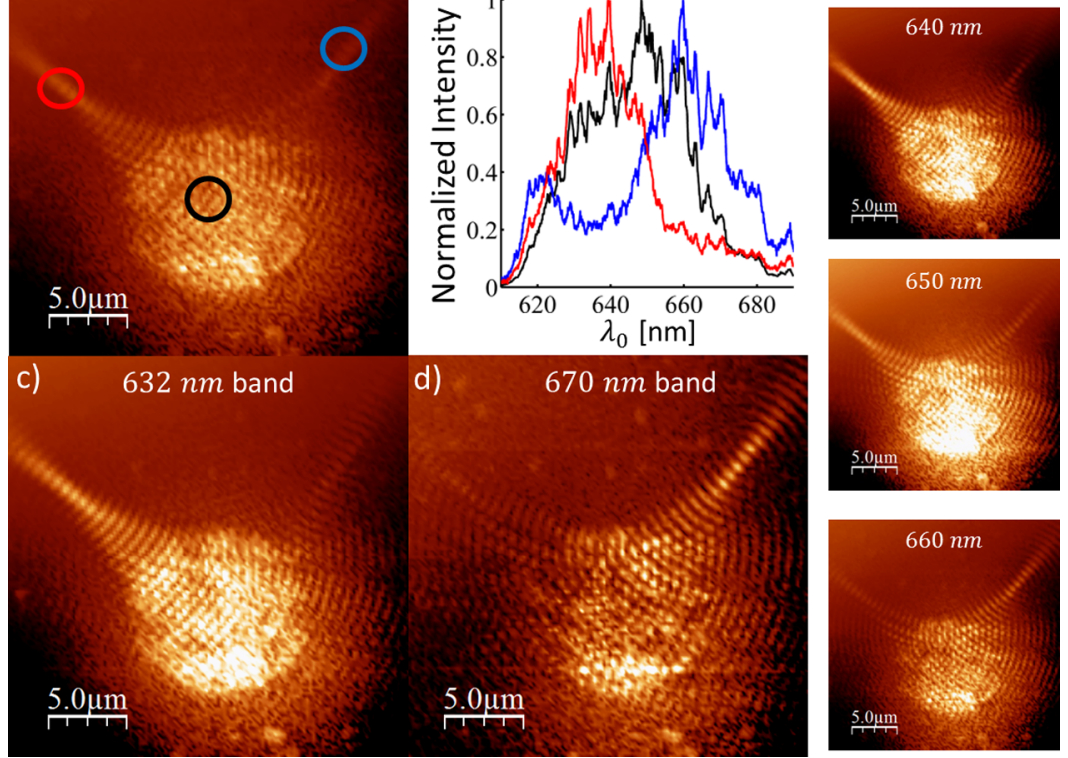

Figure 3. Spectrally resolved NSOM imaging. A single NSOM scan is performed while the metalens is illuminated with $\lambda_{0}=580-700 \mathrm{~nm}$. The same experimental setup as in Figure 2a is used except the optical fiber connected to a spectrometer. (a) Spectrally resolved NSOM image for the whole wavelength range; each pixel in the image represents the number of counts in a wavelength range of 580-700 nm (the full band). The colored circles in (a) correspond to the physical location on the metalens where the colored spectra (b) are taken, that is, the black spectrum from (b) is taken inside the black circle in (a). Each curve in (b) is normalized independently to highlight the spectral shifts. (c) Spectrally resolved NSOM image for a band of $632 \pm 3 \mathrm{~nm}$. Each pixel intensity represents the sum of all photon counts within that wavelength range. (d) Same as in panel $\mathrm{c}$ but for a wavelength range $670 \pm 3 \mathrm{~nm}$. (e) Spectrally resolved data for wavelength bands in between the operating wavelengths. A unique characteristic of the device is that the power is always concentrated into the two directions but with different relative intensities that depend on how close the wavelength is to the two operating wavelengths $(632$ and $670 \mathrm{~nm})$.

in a propagating beam of light, producing intense hot-spots instead. Furthermore, our approach allows us to choose the phase of the SPPs at the focus (relative to the incident beam), since the initial choice of point source phase is arbitrary. This can be important if a specific phase is needed, for instance, to couple the SPPs to a specific plasmonic waveguide mode or other components of a more complex circuit located at the focal positions.

Another central point of our design is to use nanoslits instead of nanoholes for coupling light to SPPs. Light passing through a nanoaperture diffracts, giving rise to a large spread of wavevectors that allows coupling into SPP modes. A nanoslit that is subwavelength predominantly in one direction reradiates SPPs preferentially when the incident light is polarized normal to the slit. Far from the nanoslit (several hundred nanometers), the SPP intensity has a simple dipole-like distribution. Nanoholes can couple free space light to SPPs, but they do so without polarization selectivity. Employing nanoslits adds polarization selectivity because light polarized perpendicular to the nanoslit couples preferentially. Horizontal nanoslits are excited by vertically polarized light and vice versa, which forms the basis for the polarization on/off selectivity and polarization tunable coupling of the metalens. Each orientation (horizontal or vertical) of nanoslits in the device has two operating free space wavelengths; vertical slits couple to $\lambda_{0}=632$ and $\lambda_{0}=670$ $\mathrm{nm}$, horizontal slits couple to $\lambda_{0}=710$ and $\lambda_{0}=750 \mathrm{~nm}$. Wavelength tunable directionality is based on the following: for a set of slits, if one of the operating wavelengths with the correct polarization is incident on the device, the only location where all of the SPPs constructively interfere is the original imaginary source point; nowhere else will all of the SPPs constructively interfere. These slits are shown in the scanning electron micrograph (SEM) of Figure 1c.

To fabricate our device, we e-beam evaporate a $50 \mathrm{~nm}$ film of gold onto a polished silicon wafer and template strip ${ }^{39}$ it onto a glass slide for decreased surface roughness. Then, after following the design depicted in Figure 1, we use focused ion beam (FIB) milling to etch the nanoslits into the gold film (Figure 1c). The sample is illuminated from below with linearly polarized light (Figure 2). The illuminating beam is at normal incidence and focused (beam waist of $\sim 10 \mu \mathrm{m}$ ) on the metalens. The incident light is emitted from a super continuum laser with a wavelength range in the band $400-850 \mathrm{~nm}$. This laser excites the nanoslits causing SPP emission while the probe of a near-field scanning optical microscope (NSOM) scans an area of the surface that includes the metalens and the focal points. Our NSOM (Nanonics Imaging Multiview) probe is a metal-coated, tapered optical fiber with a subwavelength aperture at the facet. The NSOM probe interacts with the evanescent field of the SPPs close to the metal/air interface that are then coupled into propagating modes in the optical fiber (collection mode NSOM). The other end of the fiber is connected to a single photon avalanche photodiode (SPAD) or a spectrometer. Figure 2a presents a full schematic of the experimental setup.

Since the metallic film in our device is optically thin (40-50 $\mathrm{nm}$ ), some of the incident light is transmitted through the sample. This transmitted light interferes with the SPPs at the surface such that the signal recorded by the NSOM at each pixel is the SPP intensity modulated by this interference. This useful effect allows for direct imaging of the wavefronts of the focusing SPP beam without any external reference beam or interferometric setups. Figure $2 \mathrm{~b}$ shows the operation of the 
device when illuminated by light with the correct polarization and wavelength from the laser source (one polarization and wavelength at a time). Directional focusing of SPPs on the surface is evident as the different wavelengths are focused to the four corners of a square.

In order to better understand our experimental results, we implemented an analytical model of how the designed metalens will function. We treat each nanoslit as an infinitesimal perfect electric point dipole with a direction of emission perpendicular to the slit, such that a vertical nanoslit is represented by a horizontal dipole. Adapting eq 2 from ref 18, we then sum the contribution from each antenna

$$
\begin{aligned}
E_{\text {total }}= & \sum_{i} \frac{e^{j k_{\mathrm{SPP}}\left[\left(x-x_{i}\right)^{2}+\left(y-y_{i}\right)^{2}\right]^{1 / 2}}}{\left[\left(x-x_{i}\right)^{2}+\left(y-y_{i}\right)^{2}\right]^{3 / 4}}\left[\left(x-x_{i}\right) \cos (\eta)\right. \\
& \left.-\left(y-y_{i}\right) \sin (\eta)\right]
\end{aligned}
$$

where $k_{\mathrm{SPP}}$ is the wavenumber of the surface plasmon, $x_{i} y_{i}$ is the position of the $i$ th dipole, and $\eta$ is the angle of orientation of the nanoslit relative to the vertical: 0 for vertical apertures and $\pi / 2$ for horizontal apertures. This total field is then added to the electric field produced by a flat wavefront, representing our focused Gaussian beam at the focal plane. Implementation and comparison of this analytical model to the experimental results can be seen in Figure 2b,c. The agreement between the data and the analytical model suggests that multiple reflections of SPPs from the nanoslits are not a serious factor in the performance of the device, as the model does not take this into account. Furthermore, an effective numerical aperture (NA) can be defined according to NA $=\left(\lambda_{\mathrm{SPP}} / \pi w\right)$ where $2 w$ is the full width at half-maximum of the beam at the focus. The effective NA for the metalens with this definition is calculated to be on average 0.68 for the operating wavelengths. The full width at half-maximum at the focus for all the wavelengths is roughly $\lambda_{\text {SPP. }}$. Reminiscent of the diffraction limit, this waist could be decreased by fabricating a larger metalens; a larger diameter metalens would include more wavevectors and recreate the point source more accurately. We note that the nanoslits closest to the focal point will contribute more than the nanoslits that are further, mainly due to their dipolar nature (eq 1) and their propagation losses (see Supporting Information).

In order to calculate the relative efficiency of the metalens, it is useful to compare it to an isotropic scatterer of SPPs (for example, radially or circularly polarized light incident onto a circular aperture). The percentage of SPPs contained within a given angle $\theta$ of the isotropic scatterer is simply given by $\theta$ / $360^{\circ}$. To compare this to our device, we measure the intensity of SPPs as a function of $\theta$ along the circumference of a circle with radius equal to the focal distance, $11.3 \mu \mathrm{m}$. We find that on average $23 \%$ of the SPPs at the focal radius are contained within a $10^{\circ}$ angle, which is almost an order of magnitude higher than the isotropic scatterer, which only contains $2.8 \%$ of the SPP intensity within a $10^{\circ}$ angle.

In order to further characterize our metalens, we illuminated the sample with a wide band $(580-700 \mathrm{~nm})$, chosen to include two of the operating wavelengths. In this case, the NSOM fiber is connected to a spectrometer (Andor Shamrock and Newton EMCCD Camera). The spectrally resolved NSOM data are presented in Figure 3. Interestingly, the SPPs are always concentrated into the two different channels but with different relative intensities. For example, while $\lambda_{0}=632$ and $670 \mathrm{~nm}$ light are almost completely concentrated into a single direction (following the original design), $650 \mathrm{~nm}$ light is split between two directions (Figure 3e). This behavior, imposed by the design, can be understood by noting that the difference in SPP wavelengths is small compared to $\lambda_{\mathrm{SPP}},\left(\sim 20 \mathrm{~nm}\right.$ for $\lambda_{0}=632$ and $650 \mathrm{~nm}$ light). When excited by $650 \mathrm{~nm}$ light, the apertures are only slightly offset from the equiphase lines in Figure 1a, and their emissions will constructively interfere at the source points (though not as completely as it does for 632 and 670 $\mathrm{nm}$ ). In addition, this explains why the power transfers from one direction to the other as $\lambda_{\mathrm{SPP}}$ gets closer to or further away from one of the operating wavelengths. For example, $640 \mathrm{~nm}$ light concentrates more power in the $632 \mathrm{~nm}$ channel and less into the $670 \mathrm{~nm}$ channel; however, $660 \mathrm{~nm}$ light experiences the opposite. In terms of applications, monitoring the relative intensities in each arm would allow for an on-chip spectrometer.

Figure 4 shows the focusing of the SPPs and characterizes the polarization on/off switching. In Figure 4a, the interference of
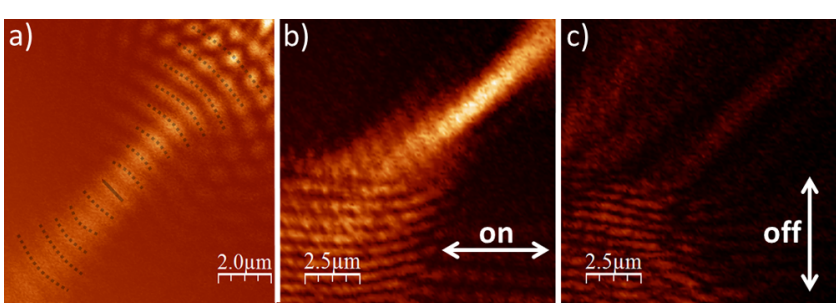

Figure 4. Focusing and polarization on/off switching. (a) NSOM image of the focal point when the metalens is illuminated with $750 \mathrm{~nm}$ light with vertical polarization. Superposed are black dashed lines that highlight the wavefront curvature as the beam focuses, with the focus denoted by the solid black line. Note the single apertures in the top right portion of the image that act as surface plasmon polariton (SPP) sources (together with all the others in the device). (b) SPP beam focusing for $\lambda_{0}=710 \mathrm{~nm}$ when the metalens is illuminated with the "on" polarization (horizontal). (c) Same as panel b but with the metalens illuminated with the "off" polarization (vertical). The ratio of the intensities at the foci of (b) and (c) are 15:1, giving $12 \mathrm{~dB}$ of modulation.

the incident beam and focusing SPPs is shown in order to view the SPP wavefronts during focusing. In Figure $4 b$, the metalens is illuminated with the polarization that maximizes coupling into SPP modes. For comparison, Figure $4 \mathrm{c}$ shows the metalens when illuminated with a polarization that is parallel to the associated nanoslits, minimizing the coupling to SPPs.

In this paper, we demonstrated a metalens design strategy that can be used to overcome some of the coupling and focusing issues for SPPs. The design strategy can be used to gain both wavelength and polarization tunability over the direction of SPP beam propagation; most importantly, by recreating the wavefront of a point source we are able to focus SPP beams after coupling. The metalens is studied under both single wavelength illumination (collecting intensity) and broadband illumination (collecting spectrally resolved intensity) with phase information in certain cases. Free space light is coupled to a nanostructured surface via subwavelength slits and steered into different directions based on its wavelength and polarization in the form of a focused SPP beam. We note that the relative efficiency of our metalens as compared to an isotropic coupler is improved by an order of magnitude. Also, the coupling conversion efficiency can be improved by optimizing the nanoslit geometry to take advantage of a plasmonic resonance. We show phase evolution of the created 
SPP beams and highlight the focusing. In principle, these beams could be easily outcoupled and reradiated back into free space modes, which would serve to completely demultiplex free space light of different wavelengths, accomplished by a single, ultrathin optical element. The design strategy presented here could open up new opportunities in the realms of integrated waveguides, on-chip spectroscopy, or broadband demultiplexers.

\section{ASSOCIATED CONTENT}

\section{S Supporting Information}

Design figures of merit, angular intensity distribution calculation, analytical calculations under varying angles of incidence, and data on a two polarization and two wavelength device are available. The Supporting Information is available free of charge on the ACS Publications website at DOI: 10.1021/acs.nanolett.5b01076.

\section{AUTHOR INFORMATION}

\section{Corresponding Author}

*E-mail: capasso@seas.harvard.edu.

\section{Funding}

The authors acknowledge support from the Harvard Nanoscale Science and Engineering Center (NSEC) under contract NSF/ PHY 06-46094, and the Center for Nanoscale Systems (CNS) at Harvard University, which is a member of the National Nanotechnology Infrastructure Network (NNIN). This work was supported by a National Science Foundation Early concept Grant for Exploratory Research (Grant ECCS-1347251), and by the Air Force Office of Scientific Research under Grant FA9550-12-1-0289.

\section{Notes}

The authors declare no competing financial interest.

\section{ACKNOWLEDGMENTS}

The authors would like to acknowledge useful discussions with R. Zhang, M. Kats, and J-P. B. Mueller. The authors acknowledge E. Orabona for the MATLAB routine used on the spectrally resolved NSOM data. The authors acknowledge Nanonics Ltd. and Andor Technology for support in the nearfield setup and spectral imaging, respectively.

\section{REFERENCES}

(1) Maier, S. A. Plasmonics: Fundamentals and Applications; Springer Science and Business Media: New York, 2007.

(2) Shalaev, V. M.; Kawata, S. Nanophotonics with Surface Plasmons; Elsevier: Oxford, 2006.

(3) Barnes, W. L.; Dereux, A.; Ebbesen, T. W. Nature 2003, 424, $824-830$.

(4) Ozbay, E. Science 2006, 113, 189-193.

(5) Yu, N.; Genevet, P.; Kats, M. A.; Aieta, F.; Tetienne, J. P.; Capasso, F.; Gaburro, Z. Science 2011, 334, 333-337.

(6) Ni, X.; Emani, N. K.; Kildishev, A. V.; Boltasseva, A.; Shalaev, V. M. Science 2012, 335, 427-427.

(7) Yu, N.; Capasso, F. Nat. Mater. 2014, 13, 139-150.

(8) Yu, N.; Aieta, F.; Genevet, P.; Kats, M. A.; Gaburro, Z.; Capasso, F. Nano Lett. 2012, 12, 6328-6333.

(9) Pors, A.; Nielsen, M. G.; Bozhevolnyi, S. I. Opt. Lett. 2013, 38, $513-515$.

(10) Ni, X.; Ishii, S.; Kildishev, A. V.; Shalaev, V. M. Light: Sci. Appl. 2013, 2, 1-5.

(11) Chen, X.; Huang, L.; Muhlenbernd, H.; Li, G.; Bai, B.; Qiaofeng, T.; Guofan, J.; Cheng-Wei, Q.; Zhang, S.; Zentgraf, T. Nat. Commun. 2012, 3, 1198-1203.
(12) Kang, M.; Feng, T.; Wang, H. T.; Li, J. Opt. Express 2012, 20, 15882-15890.

(13) Babak, M.; Mosallaei, H. Opt. Lett. 2011, 36, 2569-2571.

(14) Li, X.; Xiao, S.; Cai, B.; He, Q.; Cui, T. J.; Zhou, L. Opt. Lett. 2012, 37, 4940-4942.

(15) Pors, A.; Nielsen, M. G.; Eriksen, R. L.; Bozhevolnyi, S. I. Nano Lett. 2013, 13, 829-834.

(16) Aieta, F.; Genevet, P.; Kats, M. A.; Yu, N.; Blanchard, R.; Gaburro, Z.; Capasso, F. Nano Lett. 2012, 12, 4932-4936.

(17) Aieta, F.; Genevet, P.; Kats, M. A.; Capasso, F. Opt. Express 2013, 21, 31530-31539.

(18) Tanemura, T.; Balram, K. C.; Ly-Gagnon, D. S.; Wahl, P.; White, J. S.; Brongersma, M. L.; Miller, D. A. Nano Lett. 2011, 11, 2693-2698.

(19) Chen, Y. G.; Yang, F. Y.; Liu, J.; Li, Z. Y. Opt. Express 2014, 22, 14727-14737.

(20) Lin, J.; Mueller, J. B.; Wang, Q.; Yuan, G.; Antoniou, N.; Yuan, X. C.; Capasso, F. Science 2013, 340, 331-334.

(21) Lee, K. G.; Park, Q. H. Phys. Rev. Lett. 2005, 95, 10390211039024.

(22) López-Tejeira, F.; Rodrigo, S. G.; Martin-Moreno, L.; GarciaVidal, F. J.; Devaux, E.; Ebbesen, T. W.; Krenn, J. R.; Radko, I. P.; Bozhevolnyi, S. I.; Gonzalez, M. U.; Weeber, J. C.; Dereux, A. Nat. Phys. 2007, 3, 324-328.

(23) Liu, Z.; Steele, J. M.; Srituravanich, W.; Pikus, Y.; Sun, C.; Zhang, X. Nano Lett. 2005, 5, 1726-1729.

(24) Yin, L.; Vlasko-Vlasov, V. K.; Pearson, J.; Hiller, J. M.; Hua, J.; Welp, U.; Brown, D. E.; Kimball, C. W. Nano Lett. 2005, 5, 13991402.

(25) Gabor, D. Proc. R. Soc. London, Ser. A 1949, 454-487.

(26) Denisyuk, Y. N. Sov. Phys.-Dokl. 1962, 7, 543-548.

(27) Leith, E. N.; Upatnieks, J. J. Opt. Soc. Am. 1962, 52, 1123-1128.

(28) Cowan, J. J. Opt. Commun. 1972, 5, 69-72.

(29) Maruo, S.; Nakamura, O.; Kawata, S. Appl. Opt. 1997, 36, 2343-2346.

(30) Wang, G. P.; Sugiura, T.; Kawata, S. Appl. Opt. 2001, 40, 36493653.

(31) Genevet, P.; Capasso, F. Rep. Prog. Phys. 2015, 78, 024401024420 .

(32) Ozaki, M.; Kato, J.; Kawata, S. Science 2011, 332, 218-220.

(33) Genevet, P.; Lin, J.; Kats, M. A.; Capasso, F. Nat. Commun. 2012, 3, 1278.

(34) Dolev, I.; Epstein, I.; Arie, A. Phys. Rev. Lett. 2012, 109, 2039031-2039035.

(35) Ni, X.; Kildishev, A. V.; Shalaev, V. M. Nat. Commun. 2013, 4, 2807.

(36) Zhou, F.; Liu, Y.; Cai, W. Opt. Express 2013, 21, 4348-4354.

(37) Huang, L.; Chen, X.; Muhlenbernd, H.; Zhang, H.; Chen, S.; Bai, B.; Tan, Q.; Jin, G.; Cheah, K. W.; Qiu, C. W.; Li, J.; Zentgraf, T.; Zhang, S. Nat. Commun. 2013, 4, 2808.

(38) Orabona, E.; Ambrosio, A.; Longo, A.; Carotenuto, G.; Nicolais, L.; Maddalena, P. Opt. Lett. 2014, 39, 4263-4266.

(39) Hegner, M.; Wagner, P.; Semenza, G. Surf. Sci. 1993, 291, 3946. 